\title{
Secondary cases of meningococcal infection among close family and household contacts in England and Wales, 1984-7
}

\author{
R P D Cooke, T Riordan, D M Jones, M J Painter
}

\begin{abstract}
To determine the incidence of secondary meningococcal infection in close family and household contacts of index patients and to review the efficacy of chemoprophylaxis the records of 3256 cases occurring from 1984 through 1987 were examined. Seventeen secondary cases $(0.5 \%)$ of infection were identified among these groups. The median interval between index and secondary cases was seven weeks. Fourteen secondary cases occurred more than one week after the disease was diagnosed in the index case. Three secondary cases had not received chemoprophylaxis and in another case the infecting strain had acquired resistance to rifampicin. Prophylaxis for the close contacts of 10 out of 11 of the remaining index patients failed to fulfil all the criteria of an optimal regimen.

Even after optimal chemoprophylaxis the medical practitioner and the family should be aware of the increased and prolonged risk of secondary meningococcal infection among close contacts of patients with the disease.
\end{abstract}

\section{Introduction}

Secondary cases of meningococcal infection occur in close contacts of an index case.' Those at greatest risk are household contacts, particularly children under the age of $5 .^{2}$ Previous reports have included secondary cases occurring only within a finite period (a maximum of 60 days) after the diagnosis of the index case..$^{2.5}$ Although the greatest risk of infection occurs in the first week after recognition of the index case, ${ }^{2}$ intervals of onset between index and secondary cases of several months have been reported recently, ${ }^{6}$ suggesting that previous studies may have underestimated rates of secondary attack.

The risk of meningococcal infection in household

Public Health Laboratory Withington Hospital, Manchester M20 8LR R P D Cooke, MRCP, senior registrar in medical microbiology

T Riordan, MRCPATH, consultant microbiologist D M Jones, FRCPATH, director

\section{Manchester Town Hall,} Manchester M60 2JB M J Painter, MFCM, specialist in community medicine

Correspondence to: $\mathrm{Dr}$ R P D Cooke, Department of Microbiology, North Manchester General Hospital, Manchester M8 6RB. contacts is considered to be 500 to 800 times higher than that in the general population $(4 \cdot 2$ secondary cases per 1000 household contacts) during a non-epidemic period. ${ }^{3}$ As rifampicin may effectively eradicate the carrier state, prophylaxis with rifampicin for household contacts of a patient with meningococcal infection has been recommended in both Britain and North America. ${ }^{78}$ Yet the efficacy of chemoprophylaxis in preventing secondary meningococcal infection remains statistically unproved. ${ }^{3}$

We examined the clinical, epidemiological, and microbiological features of secondary cases of meningococcal infection in England and Wales over a four year endemic period. Because we used sensitive typing schemes to confirm that meningococci isolated in index and secondary cases were identical we could leave the maximum time interval between such cases undefined. We examined the current incidence of secondary meningococcal infection in close family and household contacts and reviewed the efficacy of chemoprophylaxis.

The criteria used for the chemoprophylaxis of meningococcal infection have been much debated. A recent report recommended that rifampicin should remain the agent of choice and be given to all close family and household contacts within 24 hours after recognition of the index case. ' Furthermore, rifampicin should be given to the index patient before discharge from hospital. Nasopharyngeal swabs should be taken from all close contacts one to two weeks after chemoprophylaxis to detect any failure to eradicate the infecting strain. We adopted these criteria as the basis of an optimal chemoprophylactic regimen against which the measures taken during this study could be compared.

\section{Patients and methods}

The study was retrospective and based on a search through computer records of meningococcal infection kept at the Manchester meningococcal reference laboratory for England and Wales from January 1984 to December 1987. Meningococcal infection was defined as $(a)$ an illness in which Neisseria meningitidis was isolated from cerebrospinal fluid or blood or (b) an illness with clinical signs of meningitis or septicaemia accompanied by a rash (macular, petechial, or purpuric ${ }^{9}$ ) in which $N$ meningitidis serogroup $\mathrm{A}, \mathrm{B}$, or $\mathrm{C}$ was isolated from the nasopharynx but not from blood or cerebrospinal fluid. During the investigation information on two clinically diagnosed index cases was provided. A secondary case was defined as meningococcal infection occurring in a close family or household contact of an index patient when the two diagnoses were made more than 24 hours apart.

The identification of $N$ meningitidis and the methods of meningococcal serogrouping, serotyping, and subtyping have been described. ${ }^{111}$ Minimum inhibitory concentrations to sodium sulphadiazine, benzylpenicillin, and rifampicin were determined for all isolates by agar dilution. Patients with identical surnames and meningococci of the same serogroup, serotype or subtype, and minimum inhibitory concentration to sulphonamide were sought. Those meningococci with serotype or subtype unidentifiable by routine coagglutination were typed by immune dot blotting. Bacterial suspensions were prepared in saline and heated to $56^{\circ} \mathrm{C}$ for 30 minutes. They were then inoculated on to nitrocellulose membrane strips, which were dried for 30 minutes at $37^{\circ} \mathrm{C}$. After soaking in $4 \%$ $\mathrm{wt} / \mathrm{vol}$ powdered skimmed milk for 30 minutes monoclonal antibodies to various class I (subtype) and class III (type) outer membrane proteins were added to the strips. After a 30 minute incubation at room temperature the strips were washed in saline and protein A-horseradish peroxidase conjugate was added. 
After a further 30 minute incubation period the strips were washed again and horseradish colour developer (Sigma) was added. A positive result was denoted by a change in colour after five minutes.

The relevant referral forms, kept at the reference laboratory, were subsequently scrutinised to confirm possible index and secondary cases by noting a common address or a familial connection between the patients. Control cases from the records were matched to the index case by age, sex, and date of onset of infection. Questionnaires on case history for the patients in the index, control, and secondary cases and their family and close contacts were prepared and sent to the relevant consultant microbiologists and medical officers for environmental health. Results were analysed with the $\chi^{2}$ test with Yates's correction or Fisher's exact test.

\section{Results}

From January 1984 to December 1987, 17 secondary cases of meningococcal infection were identified. They were associated with 16 index cases, two secondary cases having occurred in one household. Apart from four secondary cases related to an outbreak in Gloucestershire $^{6}$ there was no geographical clustering of cases. Table I shows the yearly incidence of secondary cases.

TABLE I-Yearly incidence of secondary cases of meningococcal infection, 1984-7

\begin{tabular}{lcc}
\hline Year & No of isolates received & $\begin{array}{c}\text { No of secondary cases } \\
\% \text { of total isolates/year })\end{array}$ \\
\hline 1984 & 460 & $1(0 \cdot 2)$ \\
1985 & 583 & $2(0 \cdot 3)$ \\
1986 & 1017 & $8(0 \cdot 8)$ \\
1987 & 1196 & $6(0 \cdot 5)$ \\
\hline Total & 3256 & $17(0 \cdot 5)$ \\
\hline
\end{tabular}

\section{MENINGOCOCCI}

For each index and secondary case in which culture yielded a positive result the meningococcal strains were shown to be identical. Table II shows the distribution of meningococcal serogroups among the secondary and control cases; there was no significant difference between the two groups. A meningococcus resistant

TABLE II-Serogroups of meningococci isolated in secondary and control cases, $1984-7$

\begin{tabular}{lccc}
\hline & \multicolumn{3}{c}{ Meningococcal serogroup } \\
\cline { 2 - 4 } & A & B & C \\
\hline Secondary cases $(\mathrm{n}=17)$ & 2 & 13 & 2 \\
Control cases $(\mathrm{n}=16)$ & 2 & 8 & 6
\end{tabular}

to rifampicin (minimum inhibitory concentration $>100 \mathrm{mg} / \mathrm{l}$ ) and with reduced susceptibility to benzylpenicillin (minimum inhibitory concentration $0.8 \mathrm{mg} / \mathrm{l}$ ) was grown from the cerebrospinal fluid of a "secondary patient," who had received one course of rifampicin from a hospital doctor and then another from a general practitioner. An identical, though fully sensitive, meningococcus had been isolated from the index patient's nasopharynx before chemoprophylaxis. The patient responded well to intravenous benzylpenicillin. All other meningococcal isolates were sensitive to rifampicin (minimum inhibitory concentration $<0.25 \mathrm{mg} / \mathrm{l}$ ) and benzylpenicillin (minimum inhibitory concentration $\leqslant 0 \cdot 16 \mathrm{mg} / \mathrm{l}$ ).

\section{INDEX AND SECONDARY CASES}

Clinical details were available on 14 index patients and 15 secondary patients. The index group comprised 13 children and one adult (six male, eight female). The secondary group comprised 13 children and two adults (nine male, six female). Twelve of the index children and 10 of the secondary children were aged under 7 . The median age of both groups was 3 . In the secondary group 11 of the children were siblings (seven brothers (one twin) and four sisters (one triplet)). The other two secondary children were a cousin and a close friend of the index patient. The two adult secondary patients were parents of index patients.

Meningococcal meningitis was diagnosed in all cases. Presenting symptoms and signs were similar in both index and secondary cases. No patient had a history of meningitis or tonsillectomy. There was one death, of a secondary patient, who died on admission. Analysis of the questionnaires on case history showed no significant difference in epidemiological features between the index and control cases (table III).

TABLE III-Results obtained with questionnaire on case history in index and control cases of meningococcal infection

\begin{tabular}{lcc}
\hline Epidemiological feature & $\begin{array}{c}\text { Index cases } \\
(\mathbf{n}=14)\end{array}$ & $\begin{array}{c}\text { Control cases } \\
(\mathrm{n}=14)\end{array}$ \\
\hline $\begin{array}{l}\text { Median No in household } \\
\text { Twins or triplets }\end{array}$ & 5 & 4 \\
$\begin{array}{l}\text { Respiratory symptoms within two } \\
\text { weeks before meningococcal infection }\end{array}$ & 2 & \\
$\begin{array}{l}\text { Attendance at playgroup or primary } \\
\quad \text { school }\end{array}$ & 4 & 4 \\
$\begin{array}{l}\text { Passive smoking } \\
\text { Shared bedroom }\end{array}$ & 7 & 5 \\
Shared bed & 7 & 5 \\
& 4 & 6 \\
\end{tabular}

In one secondary case the patient developed meningococcal infection while taking rifampicin prophylactically ( $N$ meningitidis cultured from the nasopharynx and cerebrospinal fluid was sensitive to rifampicin). In three secondary cases the patients did not receive chemoprophylaxis before their infection: in "ne case the patient was not born when prophylaxis was prescribed (index case 13); in the second the patient lived at a different address from the index patient and rifampicin was given only to those who slept in the index household (index case 10); and in the third no close contact received prophylaxis (index case 4). Table IV shows the time intervals between the onset of the disease in index and secondary cases.

TABLE IV - Time intervals between onset of meningococcal infection in index and secondary cases

\begin{tabular}{lcc}
\hline & \multicolumn{2}{c}{ Secondary cases $(\mathrm{n}=17)$} \\
\cline { 2 - 3 } Time interval (weeks) & No $(\%)$ & Cumulative frequency $(\%)$ \\
\hline$\leqslant 1$ & $3(17)$ & 17 \\
$2-4$ & $5(29)$ & 46 \\
$5-8$ & $2(12)$ & 58 \\
$9-12$ & $1(6)$ & 64 \\
$13-16$ & $1(6)$ & 64 \\
$17-20$ & $1(6)$ & 70 \\
$21-24$ & $1(6)$ & 76 \\
$25-28$ & $2(12)$ & 82 \\
$29-32$ & $1(6)^{\star}$ & 94 \\
$\geqslant 33$ & & 100 \\
\end{tabular}

^Interval to onset was 39 weeks.

\section{ASYMPTOMATIC CLOSE FAMILY AND HOUSEHOLD} CONTACTS

Table V shows the preventive measures used for the index patients and their close family and' household contacts. Prophylaxis for the close contacts of 13 out of 14 index patients failed to fulfil all the criteria of an optimal regimen. Contacts of only six index patients received rifampicin within 24 hours after the patients' admission to hospital. One family never received chemoprophylaxis (index case 4). The regimen for the dose of rifampicin used for all close contacts was $600 \mathrm{mg}$ (adults and children over 12), $10 \mathrm{mg} / \mathrm{kg}$ 


\begin{tabular}{lcccc}
\hline $\begin{array}{l}\text { Index } \\
\text { case }\end{array}$ & $\begin{array}{c}\text { Rifampicin given to } \\
\text { index patient before } \\
\text { discharge from } \\
\text { hospital }\end{array}$ & $\begin{array}{c}\text { Rifampicin given to } \\
\text { all close contacts }\end{array}$ & $\begin{array}{c}\text { Rifampicin given to } \\
\text { close contacts within 24 } \\
\text { hours after admission } \\
\text { of index patient }\end{array}$ & $\begin{array}{c}\text { Nasopharyngeal swabs } \\
\text { taken from close } \\
\text { contacts after } \\
\text { prophylaxis with } \\
\text { rifampicin }\end{array}$ \\
\hline 1 & - & + & + & - \\
2 & + & + & - & - \\
3 & + & + & - & - \\
4 & + & + & + & - \\
5 & + & + & + & - \\
6 & + & + & - & - \\
7 & + & + & - & - \\
8 & - & - & - & - \\
9 & - & + & + & - \\
10 & - & + & + & - \\
11 & + & + & + & + \\
12 & + & + & & - \\
13 & + & + & & - \\
14 & + & + & & - \\
\hline
\end{tabular}

(children aged 1-12), or $5 \mathrm{mg} / \mathrm{kg}$ (infants aged 3-11 months) twice daily for two days.

\section{SECONDARY ATTACK RATE AMONG CLOSE CONTACTS}

In 1984-7 the overall yearly incidence of meningococcal disease in England and Wales (population about 50 million) was $1 \cdot 6 / 100000$ inhabitants. The median number of household members in the control case group during these four years was four. Hence an estimated 9717 people were exposed to 3239 primary cases. The 17 secondary cases, which all occurred within nine months of their index cases, represent a yearly rate of secondary attack of $2 \cdot 3 / 1000$ household members, a relative risk of 144 compared with the general population.

\section{Discussion}

Despite chemoprophylaxis the incidence of secondary cases documented in this survey was high, particularly as not all cases are reported. A direct comparison with estimates in previous studies, in which no chemoprophylaxis was given, would be invalid as the definitions of a secondary case were different. One of our most striking findings was the extended interval between illness in the index and some secondary cases. The median interval of seven weeks falls beyond the limit set in some studies. ${ }^{3.5}$ The prolonged period over which family contacts are at risk clearly implies either that the initial preventive measures are failing to eradicate the infecting strain from the family or that the strain is being reintroduced from a carrier outside the family group.

Three factors may have accounted for the failure of preventive measures: the nature of the family, the extent of the prophylaxis given, and the infecting strain. By having matched control cases we could compare factors such as prior respiratory illness, passive smoking, size of family, and sharing of beds. No obvious risk factors emerged from this, though, interestingly, two of the secondary cases occurred among twins or triplets and in four of the secondary cases the patients regularly or occasionally shared a bed with another child in the family.

In one of the secondary cases the patient, who had received two courses of rifampicin after the notification of the index case, was infected with a variant of the index strain that was resistant to rifampicin. Resistance to rifampicin among meningococcal isolates associated with septicaemia or meningitis received by the Manchester meningococcal reference laboratory remains extremely low. Meningococcal disease caused by a rifampicin resistant organism in a contact who had received rifampicin prophylaxis has been reported previously. ${ }^{12}$ The current case was unusual in that the strain was also fairly insensitive to penicillin (minimum inhibitory concentration $0.8 \mathrm{mg} / \mathrm{l}$ ). The other case of interest was one in which the patient developed meningitis due to a meningococcus sensitive to rifampicin while receiving rifampicin.

When assessing the adequacy of the chemoprophylactic measures used, establishing precise details about variables such as compliance is clearly extremely difficult in a retrospective study. In one family, however, there was a complete failure to give prophylaxis, probably because of poor communication. Rifampicin was used as the prophylactic agent in all the other families and was prescribed for all the family members as recommended, though poor compliance was documented in one family. In 13 families, however, other possible shortcomings were identified. In eight cases the index patient was not given rifampicin before discharge and so may have reintroduced the strain to the family. In eight families prophylaxis was not given within 24 hours after admission of the index patient, though if the dates of onset in the secondary cases are considered the fairly short delay (less than three days in all cases) was probably not important. Because of the uncertainties about the ability of rifampicin to eradicate meningococcal carriage among a family group ${ }^{13}$ follow up swabs should be obtained about one week after prophylaxis to try to identify failures. This measure, which admittedly is rather insensitive, was performed in only two of the families in which secondary cases occurred.

The distribution of serogroups of the meningococci isolated in the secondary cases (table II) was different from that in the control case group, which in turn matched that in the general population (A 4\%, B 59\%, C $35 \%$ ). Although this difference was not significant, possibly the underrepresentation of group $C$ strains was a function of the impact of chemoprophylaxis on the higher rates of carriage and transmissibility associated with group $C$ infection compared with the more prolonged and lower rates of carriage seen with group B disease. ${ }^{6}$

Our results suggest that some of the secondary cases identified could have been prevented by a more rigorous adherence to the recommendations for chemoprophylaxis, including giving prophylaxis to the index patient before discharge and adopting swabbing after prophylaxis. We also conclude, however, that with the currently available prophylactic agents, even when administered in what is regarded as the optimal manner, the risk of infection in family contacts remains. This emphasises the need to combine chemoprophylaxis with clinical surveillance of the family group and to keep the possibility of meningitis in mind when non-specific febrile illnesses occur in the family, even months after the illness of the index patient.

We thank Mrs Joan Eldridge for her help in typing the meningococcal strains, and the specialists in community medicine and consultant microbiologists who answered the questionnaires.

1 Wall RA. The chemoprophylaxis of meningococcal infection. $\mathcal{I}$ Antimicrob Chemother 1988;21:698-700.

2 De Wals P, Hertoghe L, Borlee-Grimee I, et al. Meningococcal disease in Belgium. Secondary attack rate among household, day care nursery and preBelgium. Secondary attack rate among household, day care
elementary school contacts. I Infect 1981;3(suppl 1):53-61.

3 Meningococcal Disease Surveillance Group. Analysis of endemic meningococcal disease by serogroup and evaluation of chemoprophylaxis. I Infect Dis 1976;134:201-4.

4 Kaiser AB, Hennekens CH, Saslaw MS, Hayes PS, Bennett JV. Seroepidemiology and chemoprophylaxis of disease due to sulphonamideresistant Neisseria meningitidis in a civilian population. $\mathcal{f}$ Infect Dis 1974; 130:217-24

5 Munford RS, Taunay AE, de Morais JS, Fraser DW, Feldman RA. Spread of meningococcal infection within households. Lancet 1974;i:1275-8.

6 Stuart JM, Cartwright KAV, Jones DM, et al. An outbreak of meningococcal disease in Stonehouse: planning and execution of a large scale survey. Epidemiol Inf 1987;99:579-89.

7 Centers for Disease Control. Meningococcal disease - United States. MMWR 1981;30:113-5.

8 Public Health Laboratory Service Communicable Diseases Surveillance Centre. Report. Br Med f 1986;293:1293-4. 
9 Baxter P, Priestley B. Meningococcal rash. Lancet 1988;i:1166-7.

10 Eldridge J, Sutcliffe EM, Abbott JD, Jones DM. Serological grouping of meningococci and detection of antigen in cerebrospinal fluid by coagrlutination. Med Lab $S$ ci 1978:35:63-6.

11 Danielson D, Olcen P. Rapid serotyping of groups A, B and C meningococci by rocket-line immunoelectrophoresis and co-agglutination. $\mathcal{F}$ Clin Pathol 1979;32:136-42.

12 Cooper ER, Ellison RT III, Smith GS, et al. Rifampicin-resistant meningococcal disease in a contact patient given prophylactic rifampicin. $\mathcal{F}$ Pediat 1986;108:93-6.
13 Broome CV. The carrier state: Neisseria meningitidis. $\mathcal{f}$ Antimicrob Chemother 1986;18(suppl A):25-34.

14 Judson FN, Ehret JM. Single dose of ceftriaxone to eradicate pharyngeal Neisseria meningitidis. Lancet 1984; ii: 1462-3.

15 Gaunt PN, Lambert BE. Single dose ciprofloxacin for the eradication of pharyngeal carriage of Neisseria meningitidis. I Antimicrob Chemother 1988;21:489-96.

(Accepted 22 December 1988)

\title{
Incidence of insulin dependent diabetes in England: a study in the Oxford region, 1985-6
}

\author{
P J Bingley, E A M Gale
}

\section{Abstract}

Objective-To determine the incidence of insulin dependent diabetes mellitus up to the age of 21 in a geographically defined population in England with independent validation of completeness of case ascertainment.

Design-Prospective registration of newly diagnosed cases supplemented by centralised hospital discharge records and death certificates. Validation of ascertainment from general practitioners.

Setting-Oxford Regional Health Authority area (population 2.4 million).

Patients-All patients with insulin dependent diabetes diagnosed below age 21 during 1985-6 and resident in the region at the time of diagnosis.

Interventions-None.

End point-Validation of a method of case ascertainment for assessing temporal variation in incidence of insulin dependent diabetes.

Measurements and main results-The overall yearly incidence of newly diagnosed insulin dependent diabetes mellitus in people under 21 was 15.6 cases $/ 100000$ (95\% confidence interval 13.6 to 17.6$)$. Among males the incidence was 16.8 cases (14.0 to 19.7)/100 000 and among females 14.3 cases (11.6 to $17 \cdot 1) / 100000$. The highest incidence, in the $10-14$ year age group, was $26.4(20.9$ to 31.8$)$ new cases/ 100000 population yearly. Case ascertainment was greater than $95 \%$.

Conclusions - The incidence of insulin dependent diabetes in England is considerably higher than reported from large scale studies. It is consistent with described patterns of geographical variation. The figures provide a baseline for assessing temporal change.

\section{Introduction}

Despite the fact that insulin dependent diabetes mellitus is one of the commonest chronic illnesses of childhood and still carries considerable morbidity and mortality, little epidemiological information is available on its occurrence in England. There are, for example, no large scale studies with case ascertainment complete enough to permit comparisons with other countries in respect of overall incidence or the characteristics of patients developing the disease. Similar comparisons have shown pronounced geographical variation - for example, a child in Finland is 17 times more likely to develop insulin dependent diabetes than one in Hokkaido, Japan ${ }^{1}$-and seem likely to contribute important insights into the aetiology of the disease. Our aim was to derive accurate incidence rates for insulin dependent diabetes in a geographically defined population in England, by using independent

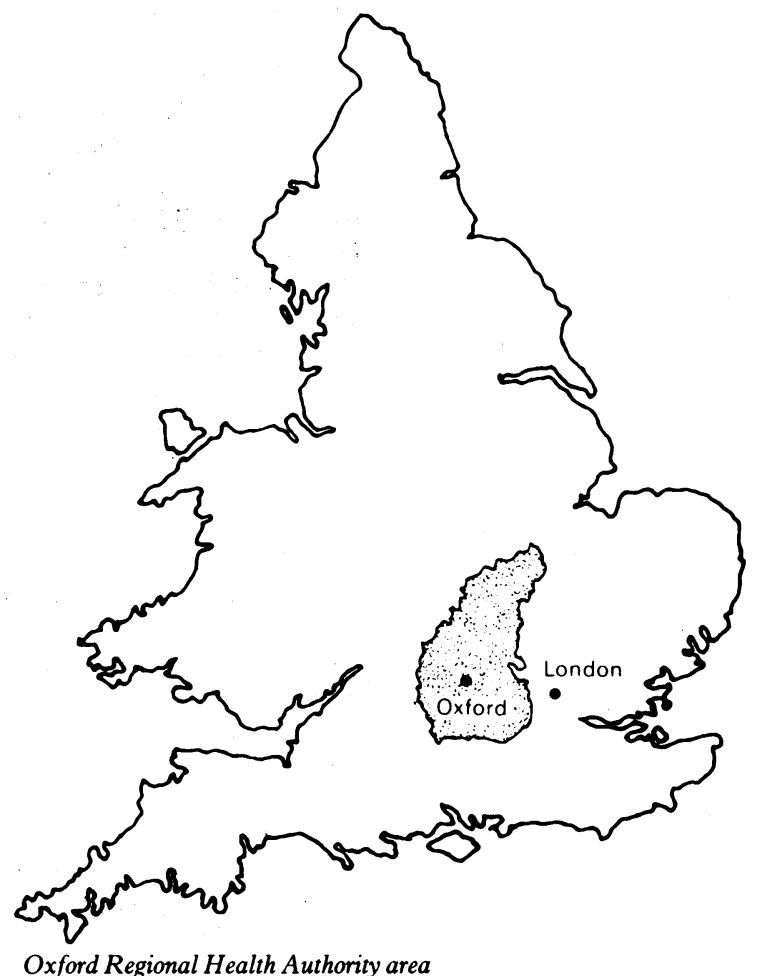

validation methods to assess the degree of case ascertainment.

Recent reports suggest that there has been a rapid increase in the incidence of insulin dependent diabetes over the past 20 to 30 years. This survey was therefore planned as a baseline for assessing the temporal variation in England. Such a study requires case ascertainment methods that will remain accurate and appropriate for the foreseeable future. We have therefore investigated the current management of new cases of insulin dependent diabetes, in particular the recent trend towards outpatient care, in assessing possible methods of ascertainment.

\section{Patients and methods}

The study formed part of the Barts-Oxford study of childhood diabetes, which covers the Oxford Regional Health Authority area (figure). This is divided into eight health districts, each containing one or more general hospitals. The area is 3130 square miles $(8107$ $\mathrm{km}^{2}$ ) and has a population of $2 \cdot 4$ million, including 750000 under the age of 21 . Six per cent of the population under 15 is thought to be non-Europid, mainly originating from the Indian subcontinent. The Barts-Oxford study is being undertaken in col- 